\section{Optical Camera Communication System for Internet of Things based on Organic Light Emitting Diodes}

P. Chavez-Burbano, S. Vitek, S. R. Teli, V. Guerra, J. Rabadan, R. Perez-Jimenez, and S. Zvanovec

The inclusion of organic light emitting diodes (OLED) in high-end devices, such as TVs and smart-phones, along with the insertion of cameras embedded in daily use devices, provides the opportunity to establish optical camera communication (OCC) systems based on OLED emitters for Internet of Things (IoT). This paper presents an experimental demonstration of this OLED based OCC system for IoT. The results suggest that despite the low emitted power of OLED devices, The resuls suses long range links can be outdoor and two ested, validating BER below 1 and give just $3.56 \cdot 10^{-3}$ for long range links.

Introduction: Nowadays, organic light emitting diodes (OLED) have been massively introduced within both the Solid State Lighting (SSL) and the high-resolution displays markets due to their low power consumption and cost-efficient manufacturing process. Worldwide companies such as Samsung, LG, Apple, and Sony, have been integrating OLEDs in their high-end smartphones (i.e. Samsung Galaxy S7, iPhone X), high-end TVs (i.e. Sony A1, LG OLED65B7V) and flexible decorative lamps.

OLEDs have been proposed as emitters for implementing Visible Light Communication (VLC) systems and for future 5G systems [1] Haigh et al. presented an OLED-based implementation using On-Off Keying (OOK) at $250 \mathrm{kbps}$ with a Bit Error Rate (BER) of $10^{-6}$ [2]. This system was improved by using pre-equalization and Multiple Input Multiple Output (MIMO) (2 emitters and 9 receiver sections), increasing the data rate up to $1.8 \mathrm{Mbps}$ [3]. In [4], a $54.9 \mathrm{Mbps}$ OLEDbased system was introduced. The authors used a high performance Artificial Neural Network (ANN) equalizer for classifying the input signal based on a training sequence for an RGB polymer LED. In the same way, Orthogonal Frequency Division Multiplexing (OFDM) has been proposed for increasing the data rate of OLED-based VLC systems. In [5], this modulation was combined with power allocation in order to achieve $10 \mathrm{Mbps}$ using a $350 \mathrm{kHz}$ bandwidth emitter. Chen et al. demonstrated a $51.6 \mathrm{Mbps}$ transmission with a BER of $3.8 \cdot 10^{-3}$ by implementing Offset Quadrature Amplitude Modulation (OQAM) [6]. However, these high-rate solutions were reached at extremely short distances (below $15 \mathrm{~cm}$ ), and further experiments only achieved $1 \mathrm{~m}$ span limit. To the authors' knowledge, currently there is no literature addressing tests with OLED based VLC systems for medium and longer distances, outdoor cases, neither the use of cameras as receivers.

In this work, the idea of using optical camera communication (OCC) systems for IoT based on OLEDs, instead of other traditional VLC schemes already proposed for data transportation in Internet of Things (IoT) networks [7], is presented and experimentally validated. In this way, longer distances can be easily reached for systems that require relatively low data transmission rates (up to $1 \mathrm{kbps}$ ). Since OCC-systems can be affected by the blooming effect, different exposure times were tested in this work. High exposure times affect several pixels rows due to the overlapped scanning of rolling shutter cameras, creating visible white halos around the illuminated portions and therefore image distortion between the bits' bands.

System Description: The proposed OCC system is based on the utilization of OLED devices as transmitters and a rolling-shutter camera as a receiver. There are several types of OLED emitters that can be used. Bendable panels are implemented in rooms, on clothing or embedded into decoration appliances. OLED monitors are usually implemented as advertisement devices. Finally, OLED screens are implemented as part of electronic devices such as smartphones and tablets. The signal is modulated using OOK during the experiments. Nonetheless, its under-sampled variants in frequency, amplitude or phase can be used for avoiding possible flickering. These modulations are part of the communication modes proposed for OCC by the IEEE $802.15 .7 \mathrm{~m}$ Optical Wireless Communications Task Group [8]. At the beginning of the transmission, a calibration signal (clock-like signal) is sent in order to adjust the system's thresholds. The OLED-device transmits the modulated signal by switching between the on and off states accordingly at frequency $f_{s}$

On the receiver side, two types of cameras can be implemented: smartdevice cameras such as those embedded into watches, smart-phones, tablets or glasses, and Closed Circuit Television (CCTV) cameras such as the ones used for security and surveillance purposes. As it is shown in Fig. 1, each frame is captured by the camera at predetermined frames per second ( $f p s$ ) using $N_{y}$ pixels of vertical resolution, and stored for its corresponding processing. To speed up the processing performance, a smaller image with only the emitter's signal information is required, so the first step is the detection of the region of interest (ROI), which can be multi-target (several simultaneous emitters). The first frame is binarized using Otsu's algorithm and dilated for finding the sources as image regions, which extrema points can be extracted. The obtained coordinates that define the boundaries of the ROI are used for the image cropping. In the multi-target case, the frame is cropped in several images, one per target. Since the first frame contains the calibration signal, the histogram information of its three channels (red, green and blue) is used for calculating the corresponding threshold values by assuring that the $50 \%$ of the data belong to each bit's level. Then, the remaining frames are binarized applying these threshold values, and the obtained B/W image (binary matrix) is projected on a $1 \mathrm{D}$ vector by averaging each row. Finally, this vector is decoded using Eq. 1 which calculate the corresponding number of pixels per bit $\left(n_{b i t}\right)$ in order to acquire the transmitted data. The number of consecutive ones or zeros are divided by the estimated $n_{b i t}$. Nevertheless, since there may be sampling frequency offset, the final output takes into account the decimal part of the division, adding one bit when needed.

$$
n_{\text {bit }}=\frac{N_{y}}{f_{s}} \cdot f p s
$$

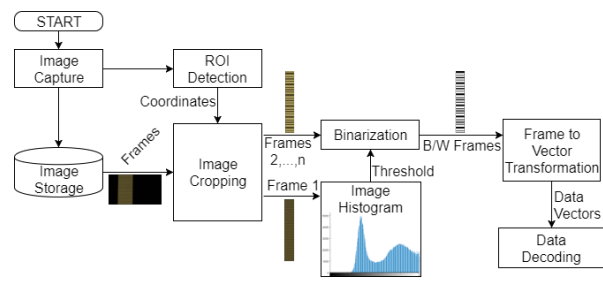

Fig. 1. OCC data processing flowchart.

To validate the proposed system, three test cases were performed at the Faculty of Electrical Engineering building of the Czech Technical University in Prague: an indoor case up to $4 \mathrm{~m}$, a corridor up to $120 \mathrm{~m}$, and finally an outdoor test up to $120 \mathrm{~m}$. In all cases, a $75 \mathrm{~lm}$ bendable bar type OLED panel was used as the emitter, with an area of $187.84 \mathrm{~mm} \times$ $37.84 \mathrm{~mm}$ and $85 \%$ of spatial luminous uniformity. This panel was switched by a MOSFET-based driver which implement an OOK non return to zero modulation. An USB rolling-shutter camera with variable exposure time (from $100 \mu \mathrm{s}$ to $30 \mathrm{~s}$ ) was selected as the receiver. This camera has a video resolution of $720 \times 540$ pixels, manual white balance of $0 \mathrm{~dB}$, gain of $3 \mathrm{~dB}$, selectable frame rate (fps), brightness and contrast of $25 / 100$, zero Hue, and saturation of 70 . For distances up to $40 \mathrm{~m}$, the camera worked with a lens of $12.5-75 \mathrm{~mm}$ and $\mathrm{F} / 1.8$. For longer indoor test outdoor tests, the camera was mounted on a 10/1000 macro telephoto lens with focal length of $1000 \mathrm{~mm}$, geometrical relative aperture $1: 10$ and angular field of view (FOV) of $2.5^{\circ}$.

At the beginning of the transmission, a calibration sequence was sent, then the data were encapsulated within 2-bytes long packets that had specific start and end delimiters (0xA sequence). In total, 1200 frames were recorded and stored per each trial. Finally, the videos were off-line processed using MATLAB routines following the procedure described in Fig. 1.

Indoor Experiment: The indoor experiment was performed within a $6 \mathrm{~m} \times 6 \mathrm{~m}$ room. The distance between the emitter and the receiver was set to $4 \mathrm{~m}$, while the switching frequency varied from $500 \mathrm{~Hz}$ to $5000 \mathrm{~Hz}$ with steps of $500 \mathrm{~Hz}$. The camera's frame rate was changed among five values: $17.5 \mathrm{fps}, 19.01 \mathrm{fps}, 25 \mathrm{fps}, 30 \mathrm{fps}$, and $50 \mathrm{fps}$; while its exposure time was set to two different values: $400 \mu$ s and $500 \mu$ s.

ELECTRONICS LETTERS 10th December 2018 Vol.00 No.00 


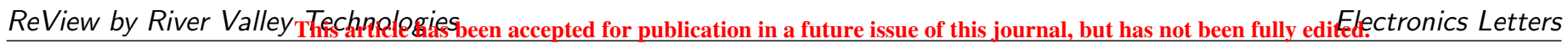
Content may change prior to final publication in an issue of the journal. To cite the paper please use the doi provided on the Digital Library page.

Fig. 2 depicts measured BER for different switching frequencies. It can be seen that the trials of $30 \mathrm{fps}$ and $50 \mathrm{fps}$ reached the best BER results, less than $10^{-2}$ for $5 \mathrm{kHz}$. Taking into account the packet encoding and the fact that each 2-byte packet was repeated twice in order to ensure its detection, the achieved data rates were approximately 120 bps and 200 bps respectively. In general, the system worked properly up to $2.5 \mathrm{kHz}$, for all the frame rates, with less than $10^{-5} \mathrm{BER}$.

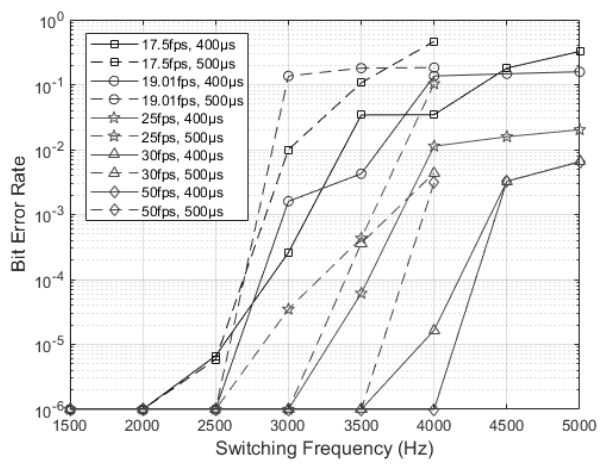

Fig. 2. BER vs switching frequency of the $4 \mathrm{~m}$-long indoor scenario.

Indoor corridor measurements: For longer indoor distances, two sets of trials were carried out in a $200 \mathrm{~m}$ corridor. In both experiments, the switching frequency was set to $1 \mathrm{kHz}$, the frame rate was set to $25 \mathrm{fps}$ and $50 \mathrm{fps}$, and the exposure time was $400 \mu \mathrm{s}$. These values were selected using Eq. 1 and considering that the target link range was longer than in the previous experiment.

In the first case, the distance ranged from $10 \mathrm{~m}$ to $40 \mathrm{~m}$ in steps of $5 \mathrm{~m}$ and the receiver used the $75 \mathrm{~mm}$ focal-length lens. The system worked as expected compared to the previous experiment, for both camera frame rates, the measured BER value remained less than $<10^{-6}$ for all cases.

In the second case, the distance varied from $20 \mathrm{~m}$ to $120 \mathrm{~m}$ in steps of $20 \mathrm{~m}$. Furthermore, the camera was mounted on the telescope, and the captured images were vertically compressed $2: 1$ to simplify the location of the emitter at the beginning of the trials. As shown in Fig 3, the BER increased from $9.68 \cdot 10^{-3}$ at $40 \mathrm{~m}$ to $5.29 \cdot 10^{-2}$ at the limit test distance $(120 \mathrm{~m})$, demonstrating that the OLED based OCC system can achieve longer distances. However, the use of the telescope may introduce misalignment errors and presented some problems related to the stability of the receiver.

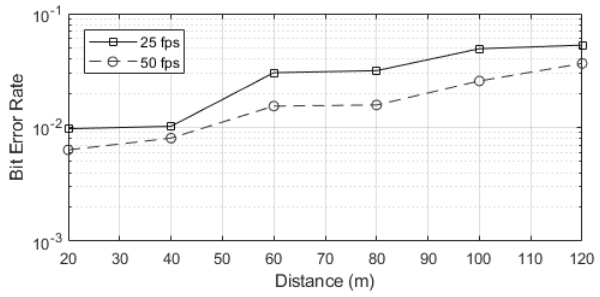

Fig. 3 Dependence of BER on distance in the case of OCC system tested within indoor corridor using a telescope at the receiver side

Outdoor measurements: Finally, the experimental tests were performed on the roof of the faculty building (approximately $30 \mathrm{~m}$ above ground) The measurements were taken in June 2018 during a scattered cloudy afternoon with the following atmospheric conditions measured by the meteorological station placed at one side of the link: temperature of $18^{\circ} \mathrm{C}$, wind speed of $9 \mathrm{~km} / \mathrm{h}$ and $60 \%$ humidity. The small wind speed and mostly the reduced link range limited the possible harmful effect of turbulence. In this experiment, two link distances were tested $(60 \mathrm{~m}$ and $120 \mathrm{~m}$ ), and the switching frequency was established to $1 \mathrm{kHz}$. The camera was mounted on the telescope with a binning of $2: 1$. In addition, the tested frame rates were $25 \mathrm{fps}$ and $50 \mathrm{fps}$, and the exposure time was $400 \mu s$.
In this case, as shown in Table 1, using a 50 fps frame rate for a distance of $60 \mathrm{~m}$, the $1 \mathrm{kHz}$ transmission achieved less than $3 \cdot 10^{-5}$ $\mathrm{BER}$, and $\mathrm{BER}$ of $3.56 \cdot 10^{-3}$ was experienced in the case of $120 \mathrm{~m}$ link.

Table 1: BER measured in the case of the outdoor scenario.

\begin{tabular}{|l|l|l|}
\hline Distance $(\mathrm{m})$ & $25 \mathrm{fps}$ & $50 \mathrm{fps}$ \\
\hline 60 & $2.77 \cdot 10^{-3}$ & $<3 \cdot 10^{-5}$ \\
\hline 120 & $6.54 \cdot 10^{-3}$ & $3.56 \cdot 10^{-3}$ \\
\hline
\end{tabular}

Conclusion: Based on the results of the different tested indoor and outdoor cases, the feasibility of an OCC system for IoT based on OLED devices was demonstrated. The indoor transmissions showed that the system can be implemented with a standard USB camera of $30 \mathrm{fps}$, obtaining BERs below $1.7 \cdot 10^{-5}$ for measurements inside a standard room ( $4 \mathrm{~m}$ ) with switching frequency up to $4 \mathrm{kHz}$, and less than $<10^{-6}$ for distances shorter than $40 \mathrm{~m}$ with $1 \mathrm{kHz}$.

These experiments also proved that the camera's exposure time influenced the decoding accuracy due to the blooming effect. Using smaller exposure time limited the overlapping read time between the rows, minimising the white halos. The exposure time of $400 \mu \mathrm{s}$ allowed an easier calibration due to the better distribution of the bands' width and improved the threshold levels' calculation and the decoding process. The laboratory tests showed that for each selected camera's frame rate, the BER's measurements are better with an exposure time of $400 \mu$ s rather than $500 \mu \mathrm{s}$.

The results shown in Table 1, verified that an outdoor OLED-based OCC system is feasible. In the same way, the number of projected pixels from the panel in the frame in the outdoor tests assured that longer distances can be easily achieved so this system can even be applied for Smart Cities. Implementations for environmental conditions monitoring, disaster detection, or smart traffic management are viable using Spatial Division Multiple Access (SDMA) allowing several emitters spatially separated within the FOV of the camera.

Acknowledgment: This work was supported in part by Escuela Acknowledgment: This work was supported in part by Escuela
Superior Politecnica del Litoral, Ecuador; by Horizon 2020 MSCA ITN project no.764461 (VISION); and by project GACR 17-17538S P. Chavez-Burbano (Facultad de Ingenieria en Electricidad y Computacion, Escuela Superior Politecnica del Litoral, Guayaquil, Guayas, Ecuador)

E-mail: paxichav@espol.edu.ec

S. Vitek, S. Teli, S. Zvanovec (Faculty of Electrical Engineering, Czech Technical University in Prague, Czech Republic)

V. Guerra, J. Rabadan, R. Perez-Jimenez (IDeTIC, Universidad de Las Palmas de Gran Canaria, Las Palmas, Spain)

\section{References}

1 Zvanovec, S., Chvojka, P., Haigh, P., Ghassemlooy, Z.: 'Visible Light Communications towards 5G', Radioengineering, 2015, 24, (1), pp. 1-9

Haigh, P. Ghassemloy, Z Rajbhandari, $S$. et al. 'Visible light communications using organic light emitting diodes', IEEE Commun. Mag., 2013, 51, (8), pp. 148-154

3 Haigh, P., Ghassemlooy, Z., Papakonstantinou, I., et al.: 'A MIMO-ANN system for increasing data rates in organic visible light communications systems', IEEE Proc. Int. Conf. on Commun., Budapest, Hungary, june 2013, pp. 5322-5327

4 Haigh, P, Bausi, F., Le Minh, H, Papakonstantinou, I., et al.: 'WavelengthMultiplexed Polymer LEDs: Towards $55 \mathrm{Mb} / \mathrm{s}$ Organic Visible Light Communications', IEEE J. Sel. Areas Commun., 2015, 33, (9), pp. 18191828

5 Le, S., Kanesan, T., Bausi, F., Haigh, P., et al.:"A $10 \mathrm{Mb} / \mathrm{s}$ visible light transmission system using a polymer light-emitting diode with orthogonal frequency division multiplexing', Optic. Lett., 2014, 39, (13), pp. 3876frequenc
3879

6 Chen, H., Xu, Z., Gao, Q., Li, S.: 'A 51.6 Mb/s Experimental VLC System Using a Monochromic Organic LED', IEEE Photon. J., 2018, 10, (2), pp. $1-12$

7 Kim, C., Koh, S.: 'Device Management and Data Transport in IoT Networks Based on Visible Light Communication', Sensors, 2018, 18, (8), pp. 2741

8 Nguyen, T., Islam, A., Yamazato, T., Jang, Y.: 'Technical Issues on IEEE $802.15 .7 \mathrm{~m}$ Image Sensor Communication Standardization', IEEE Commun. Mag., 2018, 56, (2), pp. 213-218 